\title{
Re-Visiting and Re-Coding Classic Algorithmic Art
}

\author{
André Rangel \\ Faculty of Fine Arts of the University of \\ Porto (Faculdade de Belas-Artes da \\ Universidade do Porto) \\ Av. de Rodrigues de Freitas 265 , \\ 4049-021 Porto, Portugal \\ arangel@fba.up.pt
}

\begin{abstract}
This paper and demonstration shows how the procedure of creating an electronic visualisation of some particular existing classic algorithmic artworks can give a better and deeper understanding of the original work and, as it happened in the case study, allows to find mistakes that weren't previously identified by historians, critics or researchers. This study is grounded on a case study that identified a mistake on one of the most widely spread documents that integrate Sol LeWitt's masterpiece "Variations of incomplete open cubes". In the contemporary context of digital and electronic technologies, that feed cultural superficiality, this study purposes that re-coding early algorithmic artworks can-besides being a great learning method for coding and thinking-give a deeper understanding about the thoughts and practices of the original piece authors.
\end{abstract}

Algorithmic art. Re-code. Sol LeWitt.

\section{INTRODUCTION}

The procedure of creating an electronic visualisation of existing classic algorithmic artworks gives a better and deeper understanding of the original work and, as it happened on a previous study - 3.11 - Tribute to Sol LeWitt (available at http://2017.xcoax.org/pdf/xCoAx2017-ARangel.pdf) - that grounds this proposal, it allows to find mistakes that weren't previously identified by historians, critics or researchers. During the previous study, a mistake was identified on one of the most widely spread documents that integrates Sol LeWitt's master piece Variations of Incomplete Open Cubes.

The present paper and demonstration proposal takes 3.11. - A Tribute to Sol LeWitt - an original intermedia artwork that revisits and derives from Sol LeWitt's Variations of Incomplete Open Cubes - as starting point. 3.11. - A Tribute to Sol LeWitt was designed, produced and presented with four main objectives: to concentrate the presentation of all LeWitt's 122 variations on a single electronic visualisation custom device; to actualise LeWitt's artwork through a derivation, using contemporary electronic and digital mediums unavailable to LeWitt; to emphasise the algorithmic dimension of LeWitt's work, that has been mainly acknowledged as conceptual; and above all, to develop and present a tribute to LeWitt's artwork.

LeWitt has been mainly acknowledged as a conceptual artist but, in Variations of Incomplete Open Cubes, his idea, 'machine that makes art', was an algorithm. It is verifiable that LeWitt created algorithms and this conjecture becomes evident by his own statements and practices. This idea that LeWitt created algorithms is corroborated by several authors.

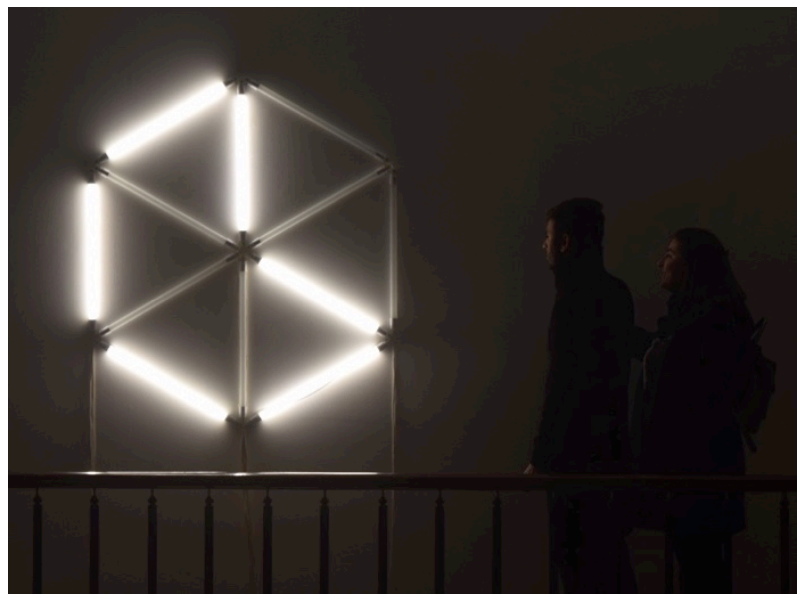

Figure 1: 3.11. - A Tribute to Sol LeWitt. Interactive Installation by André Rangel. 
In the contemporary context of digital and electronic technologies, that feed cultural superficiality, this paper propose that re-coding early algorithmic artworks can -besides being a great learning method for coding and thinking- give a deeper understanding about the thoughts and practices of the original artwork's authors.

This paper discusses how re-coding classic algorithmic artworks is related with reverse engineering by encompassing activities done to determine how the artwork itself works. The paper goes further suggesting that re-coding, beyond being a powerful learning tool that brings historic works of computer art back into the public eye and make them usable, it allows improvements, technical updates, derivations and new revelations. Video documentation of the discussed artwork is available at: https://vimeo.com/203751659 and https://vimeo.com/273101278.

\section{THE STARTING POINT: 3.11}

Early in 2017 3.11, an intermedia artwork that revisits-and derives from-Sol LeWitt's Variations of incomplete open cubes was designed, produced and presented with four main objectives: to concentrate the presentation of all 122 variations on a single electronic visualisation system; to use contemporary mediums not used by LeWitt due to their unavailability at his time; to emphasise the algorithmic dimension of LeWitt's work that has been mainly acknowledged as conceptual; and above all, to develop and present a tribute and a derivation to LeWitt's artwork.

\subsection{3 .11}

LeWitt used several media to present the different materialisations of the 122 possible variations. Wood, aluminium, photography and drawing were LeWitt's choice to present simultaneously bidimensional and tridimensional visualisations of the variations-122 sculptures, 122 photos or 122 drawings.

For 3.11., light, one Arduino microcontroller, two potentiometers, twelve analogue relays and visitors were the chosen mediums to constitute an interactive artistic experience that allows one to visualise any of the 122 LeWitt variations on the same display. 3.11. is an intermedia provocative and reactive electronic visualisation system that triggers visitors' actions, transforming them into performers, co-authors and mediums of the artwork itself. The pair of potentiometers allow humans to control the Arduino board, instructed to draw, on a display constituted by twelve T8-LED Glass tubes, any of the 122 variations of LeWitt's work. Rangel (2017) dedicated the text 3.11 - Tribute to Sol LeWitt to present and describe with detail the artwork 3.11., where he highlighted the pedagogic intentions of remembering LeWitt's work to some persons and of introducing it to others in the beginning of the XXI century.

\subsection{Sol LeWitt was an algorist}

Frieder Nake, one of the top five most acknowledge alive computer art pioneers (Franco 2020)- along side Ernest Edmonds, Manfred Mohr, Vera Molnár, and Roman Verostko-, stated that algorithmic art is the mother of conceptual art (Nake 2010). This statement grounds the proposal that LeWitt was in fact an Algorist-term apparently coined by Roman Verostko and Jean-Piérre Hébert (Nake 2012) instead of a Conceptual Artist has he has been mainly acknowledge by art historians. In Variations of Incomplete Open Cubes, his idea was an algorithm, has himself stated a 'machine that makes art'.

The proposal that LeWitt created algorithms is already well grounded on other authors and it's easily verifiable when the author himself states:

To work with a plan that is preset is one way of avoiding subjectivity. It also obviates the necessity of designing each work in turn. The plan would design the work. Some plans would require millions of variations, and so- me a limited number, but both are finite. Other plans imply infinity. In each case, however, the artist would select the basic form and rules that would govern the solution of the problem. After that the fewer decisions made in the course of completing the work, the better. This eliminates the arbitrary, the capricious, and the subjective as much as possible. This is the reason for using this method.

The words chosen by LeWitt in this description could easily be used to describe what an algorithm is. Authors like Happersett (2003), Taylor (2014), Armstrong (2016), Daudrich (2016), Rangel (2017) or Gamwell (2017) corroborate that LeWitt in fact created algorithms.

\subsection{Mistake}

During the study conducted to develop the artwork 3.11 - Tribute to Sol LeWitt, it was decided to use the results of LeWitt's Algorithm - the 122 drawings that depict 122 incomplete isometric perspectives of a cube - to feed a database that could be accessed through a custom designed interface and display. This database was stored on the nonvolatile memory space of an Arduino board. A new algorithm was designed and programmed to instruct the Arduino to retrieve each one of the 122 results as a response to the human operation of two potentiometers that integrate 3.11 interface. A more precise description of this interface can be 
found on a previous paper reporting this study 3.11 - Tribute to Sol LeWitt.

While coding the data base and the program for the Arduino board an error was noticed on the most widely known diagram of LeWitt's work: a diagram originally published in 1974 on the catalogue of the first exhibition of Variations of Incomplete Open Cubes named Sol LeWitt Incomplete Open Cubes The John Webber Gallery. This image depicts a matrix, authored by LeWitt himself, where he organises and groups the 122 structures according to their number of edges. In this matrix, the variation $8 / 15$, that should have eight edges, is represented with nine edges. Was it a mistake? During the research I did for the previous paper mentioned above, I concluded that in although LeWitt work was, posteriorly, revisited by other researchers and artists, none of them has identified the mistake.

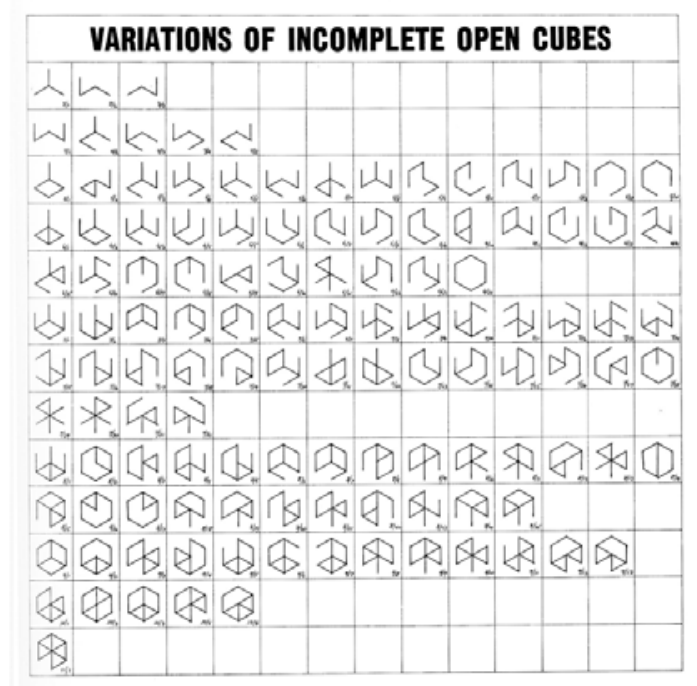

Figure 2: Sol LeWitt (1974), Diagrams for Variation of Incomplete Open Cubes, ink/pencil on paper, The John Webber Gallery, New York.

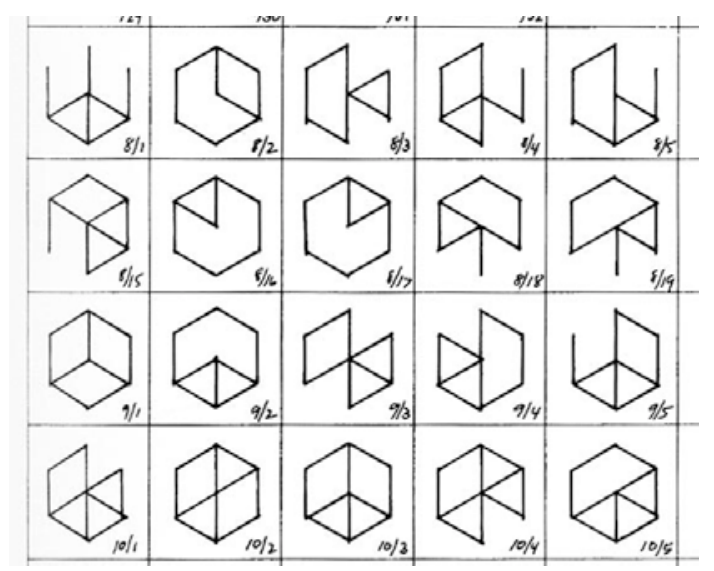

Figure 3: Detail of Figure 2 where it is visible the variation 8 / 15 with nine edges instead of eight according to Sol LeWitt's original list.

\section{RE-CODE}

Frieder Nake in is teaching activity purposes his students a recoding exercise: they observe a classic algorithmic artwork- for example a George Nees work -and they are requested to imagine how the work was achieved. In this process students have to analyse an image that results from one or several algorithmic iterations. From this analyses, students establish and order a set of rules that when executed result as an image of the same kind or style. So Nake sees coding as "the activity of describing an algorithm in the form required by some programming language" and Re-coding as "the activity of trying to re-construct the code of a drawing". Re-code (https://mepler.com/TheReCode-Project) is also the name of an online project started by Matthew Epler to "Bring historic works of computer art back into the public eye". On this website one can find several classical algorithmic artworks that were re-visited and recoded using the programming language Processing. This approach enriches the understanding one can achieve from those artworks because the result of the algorithmimage-, and its description-code- are both accessible to anyone. This approach, somehow actualises the existence of those classical artworks, in the contemporary art context, by transforming them into new media artefacts - as described by Lev Manovich in his The Language of New Media. In fact, the practice of re-coding classical algorithmic artworks opens a vast field of possibilities to adapt, transform and derivate from them into completely new artefacts. From all this possibilities, the original classical algorithmic artwork is remembered, revisited and better understood.

\subsection{Copy as learning method}

Re-coding can easily be related with the thought and practice of reverse engineering as reproduction of another manufacturer/programmer/artist production following a detailed examination of its construction, composition and production strategy. Both reverse engineering and re-code imply the deconstruction of an object, artefact or process and consequently the gaining a deep and accurate information about it.

Copying also relates to, and with, re-coding and reverse engineering. Copying was, and is, used as strategy and tool to enhance learning in several fields of knowledge. Humans need to copy when they learn how to write and to read. Copying is still an efficient strategy to develop technical skills on a wide range of human activities. Jordan and Weston (2003) about art and painting education in Japan state that "[a]t each level, student achievement is measured by the ability to replicate a technique 
flawlessly". Copy is highly antithetical in the field of art education, among other reasons, due to distinction, individuality and originality needs by artists. Shiff (1987), when discussing the antithetical method of copy as a learning method, remembers the definition of the term copy in the Dictionnaire de l'Academie des Baux-Arts - "the exact imitation of the work of a master as a means of study [...] copies are of genuine importance in the education of artists [...]" - and concludes that "individuality surfaces even the copy". So, undoubtedly, re-coding has a high pedagogical value both in terms of deepening knowledge about what classical algorithmic artists did but also in terms of skills development by the individual that re-codes. Re-coding becomes a way of appropriation of language. When someone appropriates language, they make it their own, opening new possibilities for self, individual and distinct expression.

It's worth to underline that in 3.11. the aim was not to re-code the original LeWitt algorithm that originated the 122 variations but instead, and specifically, the intention was to re-code the media display of those variations. Even by just re-coding this layer of the artwork, it was possible to discover the mistake on variation 8/15 and to design a new distinct artwork derived from LeWitt's original.

\section{CONCLUSION: CULTURAL SUPERFICIALITY}

LeWitt is an acclaimed artist since more than half a century, "Variations of Incomplete Open Cubes" is one of his major works and the mistake pointed out in the present paper occurs in LeWitt's most widely spread matrix of diagrams that depict the 122 structures. This raises an important question: how come that no one else noticed or pointed this mistake before? It seems that for decades this artwork was mainly considered on its cultural level, not on its algorithmic or technical dimensions. And even on that cultural level, the work has mainly been considered just superficially. Ommen (2016) states that "we will articulate a superficial hermeneutic as a condition of both our superficial communication environment and the superficial relationship that the public has with that environment." Art is indeed part of the environment, historians, critics and academics are simultaneously public and part of the environment. So, the answer to the question above is that, no one has noticed the mistake before because there's a tendency towards superficiality. Art history itself-grounded on Ommen-needs to be superficial "because so much of what audience experience is-the banal experience of visual surfaces in various social spaces". One could see superficial hermeneutic as a protocol of display. Superficial Art history emerges because it prefigures exemplarity, because the surfacesuperficial-differentiates artist and audience, and it emphasises technology and material. How many art historians, art critics, art lovers, art students or general public really grasped which rules constitute "Variations of Incomplete Open Cubes" algorithm and what is at stake in this classic artwork? Very, very few!

\section{REFERENCES}

Armstrong, H. (2016) Digital Design Theory: Readings from the Field. Princeton Architectural Press, New York.

Daudrich, A. (2016) Human vs. Machine: Algorithmic Methods in the Realm of Artistic Production. $x C o A x$ 2016: Fourth Conference on Computation, Communication, Aesthetics and $X$, Bergamo, 7-8 July, 75-84. Universidade do Porto, Porto, Portugal.

Franco, F. (2020) The Algorithmic Dimension: Five Artists in Conversation. Springer International Publishing, Switzerland.

Gamwell, L. (2017) Mathematics and Art: A Cultural History. Princeton University Press, New Jersey.

Happersett, S. (2003) Conceptual Art and Algorithms. Meeting Alhambra, ISAMA-Bridges 2003 Conference, University of Granada, Spain, 23-25 July 2003, 547-548. University of Granada, Granada, Spain.

Jordan, B. and Weston, V. (2003) Copying the Master and Stealing His Secrets: Talent and Training in Japanese Painting. University of Hawaii Press, Honolulu.

Manovich, L. (2001) The Language of New Media. MIT Press, Cambridge, Massachusetts.

Nake, F. (2010) Paragraphs on computer art, past and present. CAT 2010 London Conference, London, February 3, 55-63. London, UK.

Nake, F. (2012) Construction and Intuition: Creativity in Early Computer Art. McCormack, J. \& d'Inverno, M., Computers and Creativity. Springer, Berlin.

Ommen, B. (2016) The Politics of the Superficial: Visual Rhetoric and the Protocol of Display. The University of Alabama Press, Tuscaloosa, Alabama.

Rangel, A. (2017) 3.11. - Tribute to Sol LeWitt. $x$ CoAx 2017: Fifth Conference on Computation, Communication, Aesthetics and $X$, Lisbon, 6-7 July, 258-266. Universidade de Lisboa, Lisboa.

Shiff, R. (1987) Cezanne and the End of Impressionism: A Study of the Theory, Technique, and Critical Evaluation of Modern Art. University of Chicago Press, Chicago.

Taylor, Grant D. (2014) When the Machine Made Art: The Troubled History of Computer Art. Bloomsbury Academic, London. 